\title{
Parkinson's-adapted cognitive stimulation therapy: feasibility and acceptability in Lewy body spectrum disorders
}

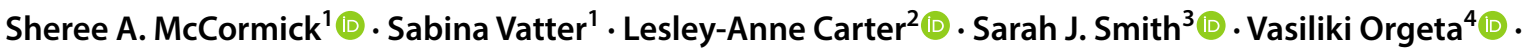

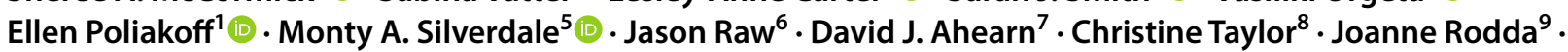 \\ Tarek Abdel-Ghany ${ }^{10} \cdot$ Benjamin Kwapong ${ }^{1} \cdot$ Iracema Leroi $^{1,11,12}{ }^{(0)}$
}

Received: 19 October 2018 / Revised: 3 March 2019 / Accepted: 7 March 2019 / Published online: 4 June 2019

(c) The Author(s) 2019

\begin{abstract}
Background Drug-based therapeutic approaches for Parkinson's disease dementia (PDD) and dementia with Lewy bodies (DLB) are moderately effective and not always tolerated. Tailoring psychosocial approaches in PDD and DLB may offer additional support and improve outcomes. We adapted home-based, care partner-delivered Cognitive Stimulation Therapy (CST) for individuals with PDD or DLB and their care partners (CST-PD).

Objectives To evaluate the feasibility, acceptability, and tolerability of CST-PD.

Methods This randomised controlled trial used mixed methods, including a process evaluation. People with PDD, DLB or mild cognitive impairment in PD (PD-MCI) and their care partners were randomised to 12 weeks of treatment as usual (TAU) or CST-PD. Outcomes were feasibility of the study conduct (i.e., recruitment, retention rate) and acceptability and tolerability of the intervention. Measures included rating scales, researcher field notes, therapy diaries, and exploratory clinical and care partner efficacy measures.

Results The recruitment target was met with 76 consenting participant-dyads. Retention in both arms was high at over $70 \%$. More than $90 \%$ of dyads undertook discrete sessions greater than 20 min duration, but the average number of sessions completed was lower than the recommended dose. Acceptability ratings (i.e., interest, motivation and sense of achievement) of the intervention were high. Participants reported no serious adverse events related to the intervention.

Conclusions The field of psychosocial interventions for PDD and DLB is newly emerging, and we demonstrated that this type of intervention is acceptable and well tolerated. Evaluating its clinical effectiveness in a full-scale randomized controlled clinical trial is warranted.
\end{abstract}

Trial registration number The trial is a psychosocial intervention with an allocated ISRCTN number 11455062.

Keywords Parkinson's disease dementia $\cdot$ Feasibility $\cdot$ Psychosocial therapy $\cdot$ Cognition $\cdot$ Quality of life

\section{Introduction}

Each year about $11 \%$ of people with Parkinson's disease (PD) develop dementia (PDD), which is likely to triple in prevalence by $2060[1,2]$. The prevalence of dementia with Lewy bodies (DLB), which encompasses around $5 \%$ of dementias overall, is also increasing [3]. Treatment

Electronic supplementary material The online version of this article (https://doi.org/10.1007/s00415-019-09329-6) contains supplementary material, which is available to authorized users.

Iracema Leroi

iracema.leroi@tcd.ie; iracema.leroi@manchester.ac.uk

Extended author information available on the last page of the article strategies for PDD and DLB have mostly focussed on medication-based interventions, such as cholinesterase inhibitors or memantine, with effectiveness of these treatments being modest at best. There is a need to find other management strategies specifically adapted for PDD, DLB and related cognitive impairment in PD (termed 'Parkinson's-related dementia'; PRD).

Cognitive Stimulation Therapy (CST) and cognitive rehabilitation therapy can enhance cognitive function, quality of life and care partner outcomes in people with non-Parkinson's-related dementias $[4,5]$ but studies in PDD and DLB are limited [6-8]. We, therefore, designed the INVEST (INdiVidualised cognitivE Stimulation Therapy) programme to develop and evaluate a personalised 
psychosocial intervention specifically for people with PRD. The first phase of INVEST involved adapting the homebased version of CST known as individualised CST (iCST; [4]), for people with PRD and their care partners [9]. Here, in the second phase, we evaluated the newly adapted therapy, CST-PD, to inform a subsequent randomised controlled trial (RCT) of effectiveness. In accordance with the UK's Medical Research Council's guidelines for the development of complex interventions, [10] our objectives were to:

(i) conduct an evaluation of the operational aspects of the study including aspects of recruitment, retention, data collection and study design;

(ii) assess the acceptability and tolerability of the intervention;

(iii) evaluate intervention fidelity, i.e., whether the intervention could be delivered, received and enacted as intended [11];

(iv) explore a possible signal of efficacy.

The first three objectives are reported here, whereas the exploratory pilot trial of efficacy (objective iv) is reported elsewhere [12].

\section{Materials and methods of the trial}

The full study protocol has been described previously [13]. Participant-dyads were recruited in the UK from four sites in Greater Manchester (primary site), and three sites in London, Derby, and Warrington. A CONSORT diagram [14] outlines the participant flow through the study, Fig. 1.

\section{Eligibility}

Participants with mild cognitive impairment in PD (PD$\mathrm{MCI})$, PDD or DLB were eligible if they were aged 18 years or older, had a diagnosis based on standard clinical diagnostic criteria [15-17] determined by the referring PD specialist, were living at home, and were on stable medication for at least 4 weeks prior to study entry. Care partners were eligible if they were 18 years or older. Capability to participate was based on clinical impression during screening and informed by scores obtained on the Unified Parkinson's Disease Rating Scale- motor examination [UPDRS-III; 18], the Schwab and England Activities of Daily Living Scale [SE-ADL; 19] and the Montreal Cognitive Assessment [MoCA; 20]. Participant-dyads were excluded if they could not understand conversational English, were non-literate, had a severe physical or psychiatric illness that precluded participation, or were taking part in another psychosocial intervention study.

\section{Study design, randomisation and ethics}

This was a single-blind parallel arm, exploratory pilot trial with an embedded feasibility study and process evaluation. An accredited clinical trials' unit undertook blocked randomisation to CST-PD, or treatment as usual (TAU), on a 1:1 participant-dyad level, without stratification. All participants provided written consent, either voluntarily or via a personal consultee declaration. Demographic data and descriptive clinical measures were collected from participant-dyads during screening. Outcome measures (see Supplementary Table 1: Outcome measure descriptions for people with Parkinson's-related dementia and care partners) were administered by assessors blind to treatment allocation. Assessors were asked to report instances of un-blinding to the study team. The study received favourable ethical opinion from the Yorkshire and The Humber-Bradford Leeds Research Ethics Committee (reference: 15/YH/0531).

\section{Study interventions}

Participants in the experimental arm were asked to complete 12 weeks (including a 2-week familiarization lead-in) of CST-PD, delivered at home by the care partner. The therapy involved participating in $30 \mathrm{~min}$ of cognitively stimulating activities, two to three times per week. The activities varied in theme and complexity and could be tailored to suit individual needs (see Supplementary Table 2: Themes and topics from the CST-PD manual). The TAU services, offered by the National Health Service in England, were available to those in the experimental arm. At the end of the intervention period, participant-dyads allocated to TAU were offered the opportunity to receive the intervention without data being collected.

\section{Assessment battery}

Clinical and care partner measures of potential efficacy, including measures of cognition, behavior, well-being and quality of life (Supplementary Table 1 and described in detail elsewhere [13]), were administered at baseline and immediately after the intervention period.

\section{Feasibility, acceptability, and tolerability evaluation framework}

To evaluate INVEST we used a modified version of the ACCEPT [21] checklist based on Thabane et al.'s guidelines for reporting feasibility trials [22]. Our modified checklist provided a systematic basis for making decisions about whether or not to accept components of our study protocol 'as is' or whether to modify it, and in what way. We applied a series of operationalized definitions of feasibility, 


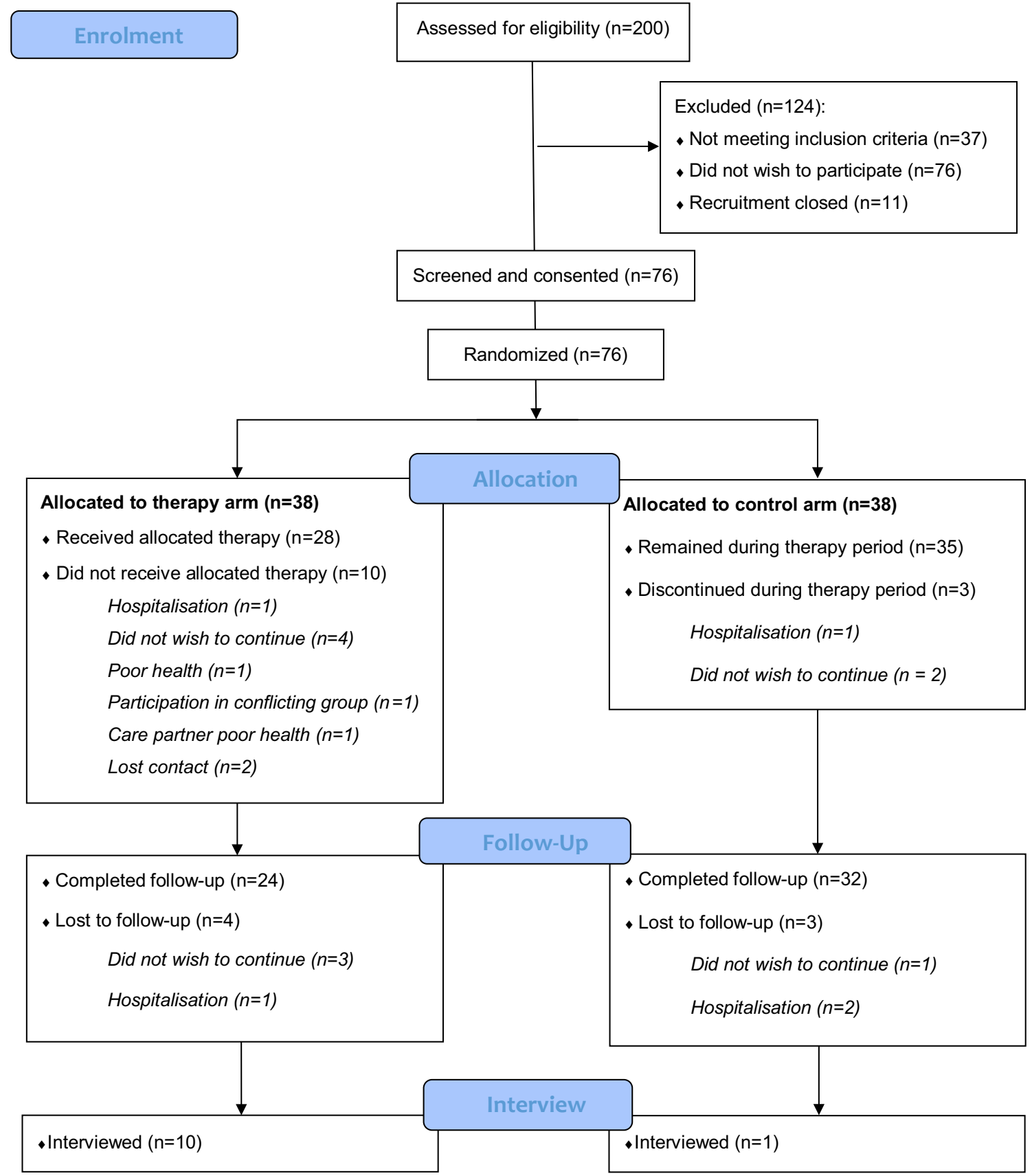

Fig. 1 CONSORT 2010 flow diagram

acceptability and tolerability, with a priori criteria, to arrive at a conclusion of 'accept', 'modify', or 'reject' (Table 2). These criteria were operationalized through a combination of pragmatic clinical judgements regarding acceptability in a population with PRD, as well as previous studies of nonpharmacological interventions for dementia and chronic mental illness [4, 30]. In particular, the 'parent' study of INVEST, a full scale RCT of iCST in people with dementia not related to Parkinson's [4], provided valuable insight into appropriate interpretations of acceptability thresholds for the different aspects of the study. The ACCEPT framework was originally designed as a flexible approach that can be applied to pragmatic clinical trials for 'real life' populations regardless of specific diagnosis [2]. Thus, its application to the current study has been appropriate and useful.

\section{Feasibility of the study procedures}

'Feasibility' was operationalised through the question, 'can it work?' [23]. Parameters included: eligibility, recruitment 
and retention rates, overall trial design (the degree to which the protocol balanced scientific and practical considerations), willingness to be randomised, blinding procedures and data collection (i.e., timing, quality, acceptability).

\section{Acceptability of the intervention}

This was defined as the extent to which the participant-dyads considered the intervention 'appropriate' [24]. We assessed care partner's perceptions of their partner's interest, motivation and sense of achievement following each therapy session. These aspects were rated in the therapy diary on a Likert-scale, consisting of the following grades: ' 1 ' $=$ strongly disagree; ' 2 ' $=$ disagree; ' 3 ' = neutral; ' 4 ' = agree; ' 5 ' = strongly agree (see Supplementary Table 3: Companion's CST-PD diary excerpt).

\section{Tolerability of the intervention}

Tolerability was defined as the ability to endure the intervention [25] and was captured by the number of serious adverse events (SAEs) related to either member of the participantdyad feeling burdened or frustrated.

\section{Intervention fidelity (delivery, receipt and enactment)}

Intervention delivery was operationalised through two questions, 'can researchers deliver therapy training to care partners?' and 'can care partners deliver the therapy to their partners?' Similarly, receipt was operationalised as, 'did care partners receive the therapy training as intended?' and 'did participants with PRD receive CST-PD as intended?' To evaluate enactment we asked, 'did participant-dyads complete the recommended amount of therapy during the intervention period?' Measures included a training checklist, a training evaluation form, a therapy skills self-assessment, and diary entries (acceptability ratings and free-text entries).

\section{Qualitative investigation}

We conducted semi-structured interviews at the end of the intervention with a purposive sample of 11 participant-dyads who undertook the therapy to gather evidence to support or refute our conclusions regarding the categorisations of 'accept', 'modify' or 'reject'. This sample size is considered sufficient to capture all emerging themes, as data saturation (rather than 'theoretical saturation') was likely to have been reached. We followed guidance suggesting that if participants are relatively homogeneous (e.g., as in our case, in which participants all fell within a single diagnostic spectrum and had a close age-range), then a purposive sample size approaching 12 will make it possible to 'render a fairly exhaustive data set' [26].

\section{Sample size}

We applied guidance on sample sizes for exploratory work $[27,28]$ and took a conservative approach, estimating the standardised effect size on cognition of the parent form of the intervention to be 0.4 , and utilizing a one-sided test and a less stringent significance level of 0.2 to avoid missing a promising effect. Thus, assuming $80 \%$ power and a correlation coefficient of 0.5 between baseline and endpoint on cognitive outcomes, the required sample size, including allowance for attrition, was 38 dyads per group at enrolment [13].

\section{Data analysis}

Quantitative data were analysed as descriptive statistics, qualitative data were analysed using thematic analysis [29]. Theme identification and initial coding were performed by authors SAM, SV and BK. The coding framework was further developed and themes defined through an iterative process by the INVEST investigators (SAM, SV, SJS and IL). To reach consensus, alternative meanings and interpretations were discussed in the context of all transcripts.

\section{Results}

\section{Feasibility of the study procedures}

Findings from all data sources regarding the feasibility aspects of the study procedures are outlined in the modified ATTEND checklist, Table 1.

\section{Recruitment/randomisation}

The recruitment rate in the first 10 months was 0.8 dyads per site per month, which was lower than expected, and likely influenced by seasonal variation and holidays. Thus, three additional sites were added to enable us to recruit to target at a rate of 0.9 dyads per site per month, approaching the 'modify' threshold of 1-2 dyads per site. Of the 200 participant-dyads referred to the study team, 37 were ineligible, 11 were referred after recruitment had ended and 76 were unwilling to participate. Reasons for ineligibility included: no diagnosis of PD or cognitive impairment in PD; distance from the study centre; being admitted to a care home; and having a medical or psychiatric condition too severe to enable study participation. Reasons for nonparticipation by eligible people included: health issues; worsening dementia and apathy in the participant; care 


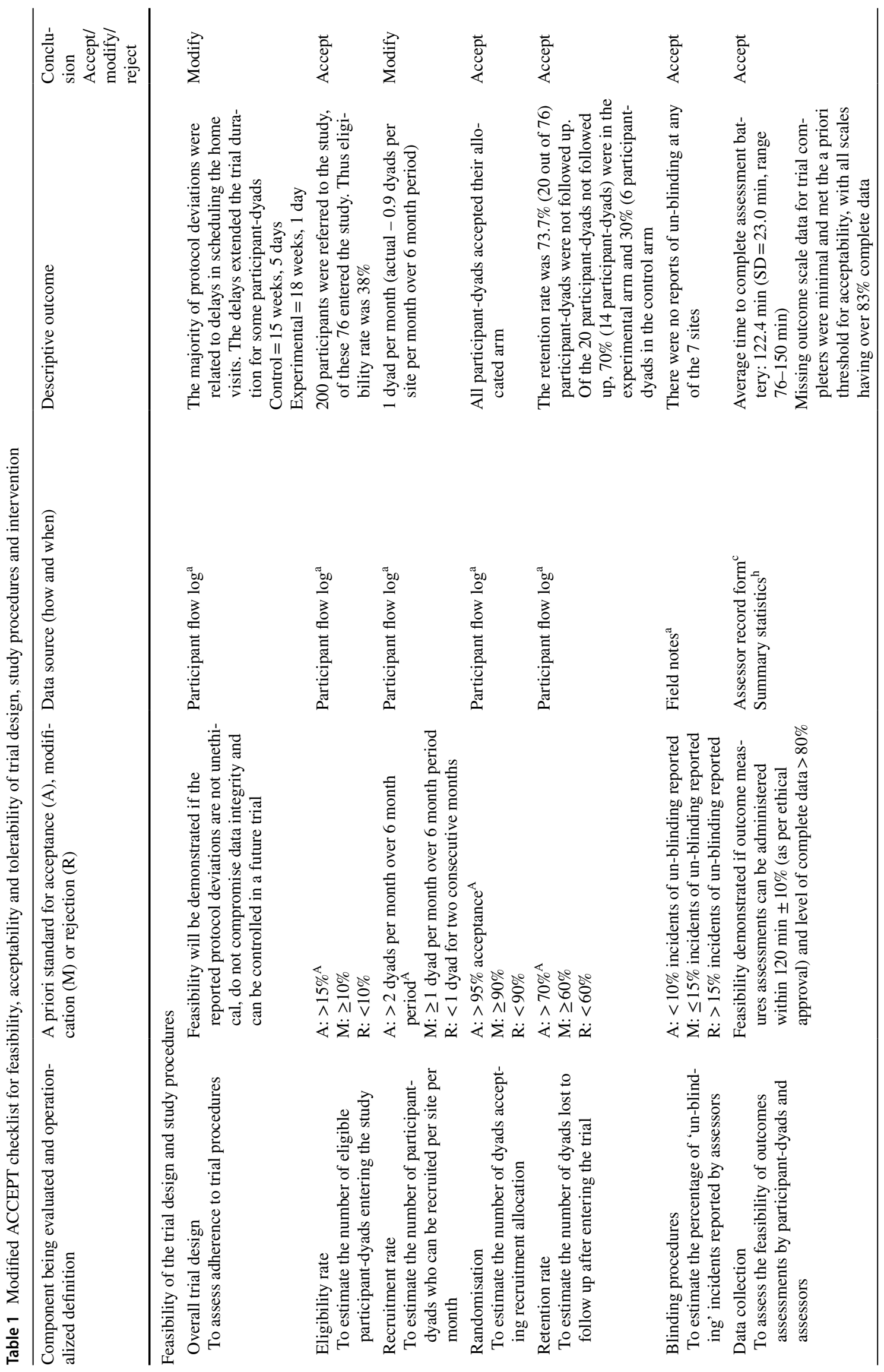




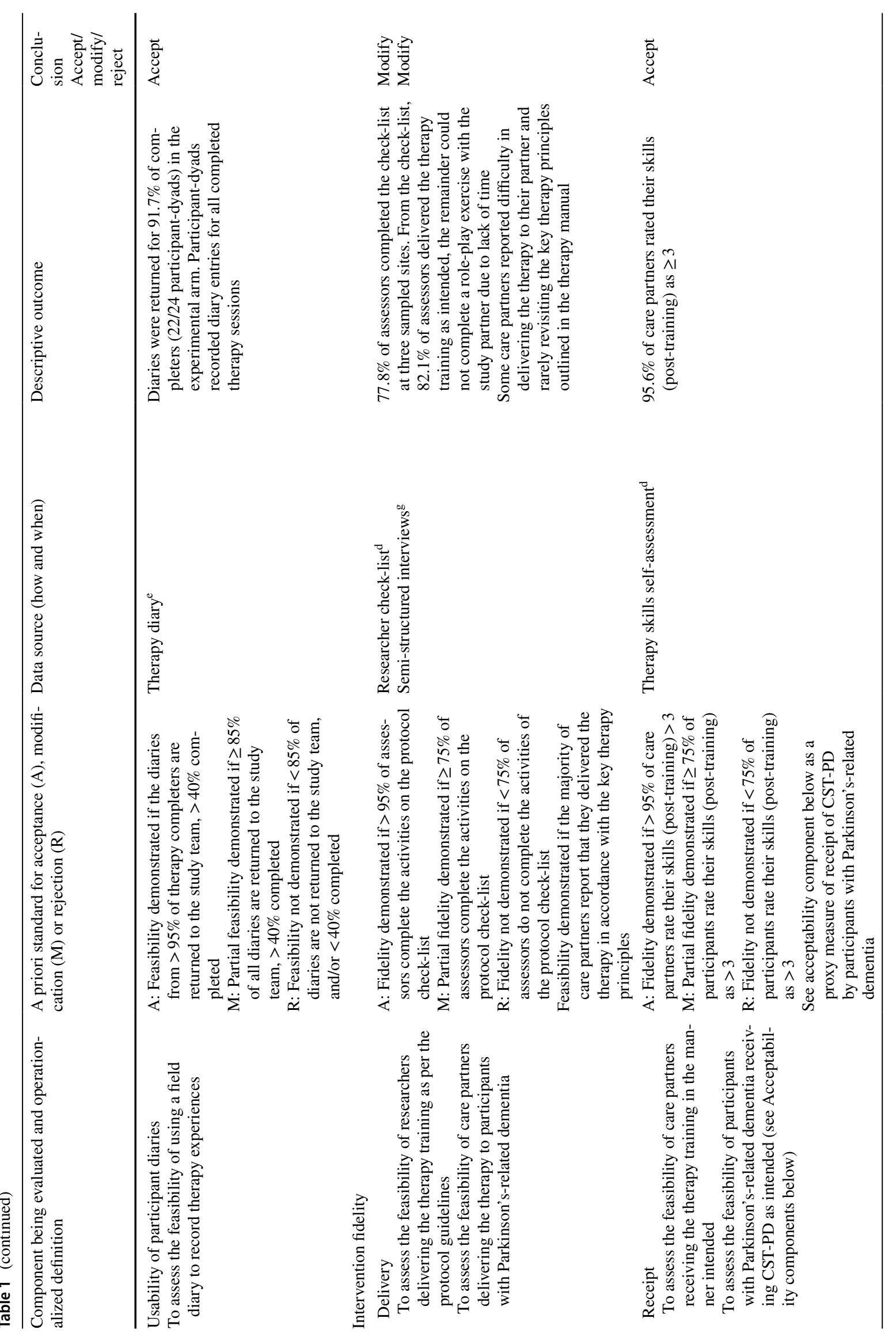




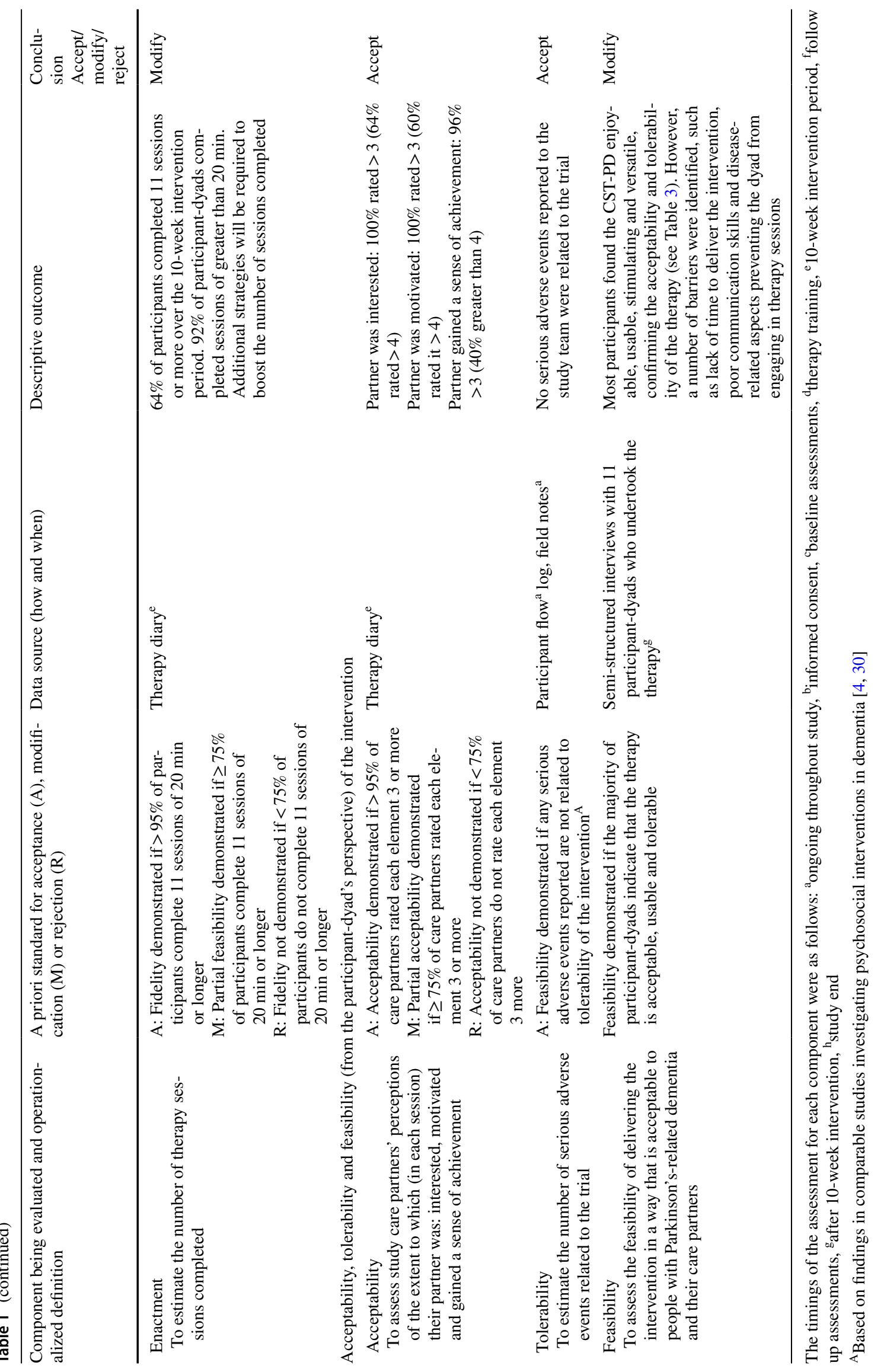


partner burden; administrative errors leading to failure to contact the dyad; and unwillingness to be randomised.

All participants $(n=76)$ had the capacity to consent to participation at the start of the trial. During the trial, four participants lost the capacity to consent and a consultee declaration form for continuation was completed by their care partners. Of the 76 participant-dyads consented, 38 were randomised to the CST-PD and 38 to TAU, none withdrew due to group allocation. These data suggest participantdyads were willing to be randomised.

Of the participants with PRD, $19.8 \%(n=15)$ had a diagnosis of PD-MCI, 52.6\% $(n=40)$ had PDD, and 27.6\% $(n=21)$ had DLB. Of this group, $79 \%(n=60)$ were male, $93.4 \%$ were white $(n=71)$ with a mean age of 74.5 years $(\mathrm{SD}=6.74)$. Of the care partners, $84.2 \%(n=65)$ were living with their study partner, $11 \%(n=8)$ were male; $77.6 \%$ $(n=59)$ were spouses or partners, $17.1 \%(n=13)$ were relatives and the remainder 5.3\% $(n=4)$ included a live-in carer, a live-in divorcee, a friend and a grandchild. Baseline demographics, per arm, are shown in Table 2. There was some variability, notably the participant-dyads in CST-PD had a higher level of schooling and a slightly higher proportion of people with PDD. The average number of years members of dyads were known to each other was slightly lower in CSTPD. This case mix imbalance suggests that randomisation was not fully effective.

\section{Eligibility criteria}

Of the 200 participant-dyads referred to the study (see Fig. 1), 76 dyads were contacted and screened for eligibility. Of these, all met eligibility criteria and were subsequently randomised. This resulted in an eligibility rate of $36 \%$, meeting the 'accept' threshold.

\section{Retention}

The retention rate was $73.7 \%$ and meets the 'accept' threshold; 56 of 76 consented participant-dyads completed the trial. Of those who were not followed up, 70\% (14 of 20) were in CST-PD and 30\% (6 out of 20) were in TAU. Out of the total consented in each inclusion group, 25\% (10 of 40) who were not followed up had PDD, 20\% (3 of 15) had PD-MCI, and 14\% (3 of 21) had DLB. Deteriorating health was the main reason for withdrawal across arms and inclusion groups. Three dyads withdrew from CST-PD as they found it difficult to discuss topics spontaneously or did not like reminiscing.

\section{Study design}

We estimated that participant-dyads would remain in the trial for 15 weeks \pm 2 weeks. This was achieved in TAU (15 weeks, 5 days), but not in CST-PD (18 weeks, 1 day). In CST-PD, the additional home-visit for therapy training introduced unforeseen delays mainly due to unavailability of participant-dyads.

\section{Blinding}

No instances of un-blinding were reported to the study team across sites.

\section{Feasibility of outcome measures}

The majority of outcome measures were feasible to administer; however, Goal Attainment Scaling (GAS [30]) was removed early in the trial as assessors reported that it was difficult to determine person-centred and measurable goals in the timeframe allocated. An early audit of the Addenbrooke's Cognitive Examination-III (ACE-III [31]) at the primary site identified inconsistencies in scoring between assessors. To improve accuracy and maintain consistency, all assessors received additional training and a scoring checklist. Missing outcome scale data for trial completers were minimal and met the a priori threshold for acceptability, with all scales having over $83 \%$ complete data. Subsequent analyses of the data revealed no differential missingness conditional on the baseline factors [13].

The therapy diary was feasible in terms of recording participant-dyads experiences; $91.7 \%$ of diaries were returned by study completers who undertook the therapy, entries were recorded for all completed therapy sessions. Qualitative investigation supported these findings, for example:

"It would have been useful to have had more room for feedback on the diary, yes, um, 'cause I'd sort of squashed quite a lot in there." [Care partner, CS17].

\section{Acceptability of the intervention}

The intervention was acceptable, based on the care partners' perceptions of the extent to which (in each session) their partner was interested, motivated, and gained a sense of achievement. All a priori targets were exceeded; at least $75 \%$ of care partners rated the aspects within the target range (see Table 1). Qualitative findings support the diary ratings, for example:

"[name] chose an article he had read in an aeroplane magazine and showed much more interest and enthusiasm in. It is a subject dear to his heart." [Care partner, CS17]. 
Table 2 Baseline demographic and clinical variables in the experimental (CST-PD) and control (TAU) arms

\begin{tabular}{|c|c|c|c|c|c|c|c|c|c|c|c|}
\hline \multirow[t]{3}{*}{ Categorical variables } & \multicolumn{6}{|c|}{ People with Parkinson's-related dementia $(n=38)$} & \multicolumn{5}{|c|}{ Care partners $(n=38)$} \\
\hline & \multicolumn{3}{|c|}{ Control } & \multicolumn{3}{|c|}{ Intervention } & \multicolumn{2}{|c|}{ Control } & & \multicolumn{2}{|c|}{ Intervention } \\
\hline & $n$ & $\%$ & & $n$ & $\%$ & & $n$ & $\%$ & & $n$ & $\%$ \\
\hline \multicolumn{12}{|l|}{ Gender } \\
\hline Female & 8 & 12.05 & & 8 & 21.05 & & 35 & 92.11 & & 33 & 86.84 \\
\hline Male & 30 & 78.95 & & 30 & 78.95 & & 3 & 7.89 & & 5 & 13.16 \\
\hline \multicolumn{12}{|l|}{ Ethnicity } \\
\hline White & 35 & 92.11 & & 36 & 94.74 & & 35 & 92.11 & & 35 & 92.11 \\
\hline Non-White & 2 & 5.26 & & 2 & 5.26 & & 2 & 5.26 & & 3 & 7.89 \\
\hline Did not specify & 1 & 2.63 & & 0 & 0.00 & & 1 & 2.36 & & 0 & 0.00 \\
\hline \multicolumn{12}{|l|}{ Education level } \\
\hline Up to 18 year old schooling & 22 & 57.89 & & 18 & 47.37 & & 20 & 52.63 & & 17 & 44.74 \\
\hline $\begin{array}{l}\text { Further education and } \\
\text { higher }\end{array}$ & 16 & 42.11 & & 20 & 52.63 & & 18 & 47.37 & & 21 & 55.26 \\
\hline \multicolumn{12}{|l|}{ Marital status } \\
\hline Single & 6 & 15.79 & & 6 & 15.79 & & 7 & 18.42 & & 6 & 15.79 \\
\hline Married/partnership & 32 & 84.21 & & 32 & 84.21 & & 31 & 81.58 & & 32 & 84.21 \\
\hline \multicolumn{12}{|l|}{ Living status } \\
\hline Alone & 5 & 13.16 & & 1 & 2.63 & & 2 & 5.26 & & 0 & 0.00 \\
\hline With others & 33 & 86.84 & & 37 & 97.37 & & 36 & 94.74 & & 38 & 100.00 \\
\hline \multicolumn{12}{|l|}{ Diagnosis } \\
\hline PD-MCI & 8 & 21.05 & & 7 & 18.42 & & & & & & \\
\hline PDD & 18 & 47.37 & & 22 & 57.89 & & & & & & \\
\hline DLB & 12 & 31.58 & & 9 & 23.68 & & & & & & \\
\hline \multicolumn{12}{|l|}{ Dyad relationship } \\
\hline Spouse/partner & & & & & & & 28 & 73.68 & & 31 & 81.58 \\
\hline Son/daughter & & & & & & & 9 & 23.68 & & 4 & 10.53 \\
\hline Other & & & & & & & 1 & 2.63 & & 3 & 7.89 \\
\hline \multicolumn{12}{|c|}{ Caregiving weekly hours (up to an average of) } \\
\hline $1 \mathrm{~h}$ per day & & & & & & & 10 & 26.32 & & 5 & 13.16 \\
\hline $8 \mathrm{~h}$ per day & & & & & & & 9 & 23.68 & & 13 & 34.21 \\
\hline $24 \mathrm{~h}$ a day & & & & & & & 19 & 50.00 & & 20 & 52.63 \\
\hline Continuous variables & $n$ & Median; IQR [range] & $n$ & Mec & ian; IQR [range] & $n$ & Median & IQR [range] & $n$ & Median; IC & [range] \\
\hline Age & 38 & $75 ; 72-81[61-90]$ & 38 & 74.5 & $0 ; 68-77$ [55-84] & 38 & $68.50 ; 5$ & $-72[43-85]$ & 38 & $67 ; 59-71$ & $1-88]$ \\
\hline Dyad known years & & & & & & 29 & $50 ; 43-$ & $6[3-68]$ & 34 & $46 ; 30-52$ & $.5-70]$ \\
\hline Caregiving years & & & & & & 38 & $2.50 ; 1-$ & {$[0-15]$} & 38 & $3.25 ; 1.5-\varepsilon$ & 0-20] \\
\hline $\begin{array}{l}\text { Montreal Cognitive Assess- } \\
\text { ment (MoCA) }\end{array}$ & 35 & $19 ; 15-22$ [7-24] & 36 & 17.5 & $15-21.5[8-30]$ & & & & & & \\
\hline Schwab-England score & 37 & $60 ; 35-80$ [10-100] & 37 & 60 & $0-70[10-90]$ & & & & & & \\
\hline UPDRS motor score & 38 & $34 ; 17.50-40.25$ [9-69] & 37 & 24 & $8-38[8-58]$ & & & & & & \\
\hline $\begin{array}{l}\text { Duration of clinical symp- } \\
\text { toms, years }\end{array}$ & 38 & $5.5 ; 2-10[0-33]$ & 38 & & $-10.50[0.5-24]$ & & & & & & \\
\hline
\end{tabular}

\section{Tolerability of the intervention}

Of the 9 SAEs reported, 5 were from CST-PD and 4 from TAU. None of the SAEs reported were due to the trial, suggesting that the intervention was well tolerated.

\section{Intervention for TAU completers}

All participant-dyads who completed their TAU arm ( $n=32$ dyads) were offered the intervention and 50\% of dyads received the intervention. Data from these 
participants, however, was not collected due to ethical constraints.

\section{Intervention fidelity}

\section{Delivery and receipt of therapy training}

The training protocol checklist revealed that $82.1 \%$ of researchers delivered CST-PD training as intended, the remainder completed all but the role-play exercise due to lack of time. The a priori target of CST-PD training being received as intended by the care partner was exceeded; after therapy training $95.6 \%$ of care partners $(n=23)$ rated their CST-PD skills within the 'accept' threshold. Furthermore, the training evaluation form, completed anonymously by care partners $(n=23)$, revealed that $95.4 \%$ found CST-PD training acceptable and $76.2 \%$ felt that they had received adequate instruction about how to deliver CST-PD.

Concerning the delivery of CST-PD by the care partner and receipt by the person with PRD, qualitative investigation revealed that some care partners struggled with personalising the activities, and/or coping with their partner's disease symptoms. For example:

- [name] took more interest in the subject today and it was less like 'pulling teeth'. [...] He seemed to enjoy the whole session more today. [Care partner, CS17].

- Have we done it right? I felt sometimes that maybe I was the problem. Maybe I wasn't approaching this right and I wasn't getting the best out of [him]. [Care partner, CS4].

It is possible that the challenges faced by some care partners may have influenced the receipt of CST-PD by the person with PRD, for example:

- You'd come to the question and you'd say, "Well, I've already said that", so what else can I say? [Participant with PRD, CS5].

That said, the acceptability ratings for interest, motivation and sense of achievement (reported above) were high, suggesting that people with PRD received the therapy in the manner intended.

\section{Enactment}

During the intervention period, $64 \%$ of participants completed 11 or more sessions, with the average number of sessions being $1.76(\mathrm{SD}=0.72)$ per week. The majority $(92 \%)$ of participant-dyads completed sessions of longer than 20 min. Thus, the 'modify' target threshold for enactment was met. The most frequently cited barriers to enactment included disease symptoms and availability. For example:
- "A barrier for you, dad, would have been two things. One that you wanted to do the session but I wasn't here to do one, so lack of availability of me, and second your health because your health varies so much day to day." [Care partner, CS9].

\section{Qualitative findings}

Eleven participant-dyads, who had completed the 10-week CST-PD protocol, were interviewed. Of these 11 dyads, one dyad completed the CST-PD intervention following their 10-week TAU participation. Of the people with PRD, six males had a diagnosis of PD-MCI, four had a diagnosis of PDD, and one had a diagnosis of DLB. The median age of participants with PRD was 72.0 (interquartile range $[\mathrm{IQR}]=68.5-77.0)$ years, median MoCA score of 21.0 (IQR = 17.0-24.5), median H\&Y stage of 3.0 $(\mathrm{IQR}=1.8-3.5)$, and median years since PD diagnosis of $8.0(\mathrm{IQR}=3.0-11.0)$ years. With respect to care partners, the sample comprised 11 females with a median age of 67.0 $(\mathrm{IQR}=65.0-70.5)$ years, of whom nine were spouses/life partners and two were adult children. The semi-structured interviews identified five themes related to overall feasibility, Table 3.

Themes supporting quantitative findings:

1. Interest/enjoyment.

2. New opportunities.

3. Mastery.

4. Lack of time/availability.

Themes refuting quantitative findings:

5. Challenges in delivering CST.

\section{Discussion}

This is the first report of a psychosocial therapy specifically adapted for people with PDD, DLB or PD-MCI and their care partners. The intervention, designed to be pragmatic, flexible and low cost, involved collaboration and cooperation between the person with PRD and their care partner. In sum, we found that undertaking a RCT of the intervention was feasible and that the intervention itself was acceptable and well tolerated by both members of the participant-dyad, although certain modifications will be needed in a future trial.

For the components for the trial which we classified as 'modify' on the ACCEPT checklist, possible modifications for a future trial would include: (1) the trial design should include more flexibility in timing of visits since arranging home visits in older, potentially frail participants often 
Table 3 Key themes emerging from the semi-structured interviews with 11 participant-dyads

Theme Quote from diary or interview

New opportunities

Interest/enjoyment

Mastery

Lack of time/availability
I think when she said we'll do it wherever in the week, so, I was pleased about that. [Participant with PRD, CS6, interview]

"When it was about topics that we were familiar with, you certainly were stimulated and excited to take part, dad, and afterwards you would be tired because you've been active for that time." [Care partner, CS9, interview]

It took a lot of pressure off us, for, you know, in as much as it gave us a fresh thing to focus on. [Care partner, CS9, interview]

Sometimes we were enjoying ourselves that much it got to $45 \mathrm{~min}$. [Care partner, CS5, interview] Time spent doing something useful. [Care partner, CS15, diary]

It's been great and to say it's, um, it deals with memories and things that I've forgotten about. [Participant with PRD, CS12, interview]

As well as me doing it and [wife], um, family and friends have asked what we're doing and it's, an interest, so we've, it gives us a chance to discuss and raise awareness. That, that's a big benefit already, plus the fact that, um, I, I firmly believe exercise is, is the key, both physical and mental, I don't do either of them enough so, you know. So, anything that comes along to my doorstep is brilliant, that's another good plus. [Participant with PRD, CS12, interview]

And there's so much choice. There's so much choice looking through the book, you know, it really is, it's quite fun deciding, "well, we'll do this" or whatever, you know. [Care partner, CS12, interview]

I loved the book. I think it's lovely, I think everybody should have one. [Care partner, CS12, interview]

It was very attractively presented I thought. The layout, was, was quite, was quite good. [...] They're all kind of like easy to look at and to follow. [Participant with PRD, CS17, interview]

I must admit I've tended to go for things with pictures rather than too many [words]. [...] The pictures are so, oh they're so nice. This one, "look at this." [Care partner, CS11, interview]

To recall some of the things we went through together, as children, as teenagers what have you, I enjoyed it. [Participant with PRD, CS9, interview]

We really enjoyed, you know, we enjoyed playing games together. [Care partner, CS12, interview] Found it easy [Care partner, CS5, diary]

As he has travelled considerably he was able to talk about this topic very well. [Care partner, CS1, diary]

He seemed to enjoy the whole session more today. [Care partner, CS17, diary]

Some of the questions [...] did require more thought. [Participant with PRD, CS17, interview]

Some of my childhood and things that had gone past, you forgotten all of that you're only assisted, until we sat down in here and we went through the sort of headlines and it was fantastic to thread those paths again and then I can compare with other people's experiences. [Participant with PRD, CS9, interview]

3 times a week that was enough, well for us it was enough because we had other things going on in our lives. [Care partner, CS9, interview]

I think our only barriers were if you weren't well or I've been busy. [Care partner, CS9, interview]

Well time was the barrier and that I don't necessary want to do more, one, I think we are more joined at the hip than we would want to be anyway so actually more intensive therapy is the last thing I want whereas when we're in a group the dynamics is very different. [Care partner, CS5, interview]

I think, the ones [activities] that we got into there wasn't enough time. [Participant with PRD, CS5, interview]

Timing was wrong for me as I was in the middle of something. [Care partner, CS4, diary]

Feeling under pressure due to time constraints. [Care partner, CS3, diary] 
Table 3 (continued)

\begin{tabular}{|c|c|}
\hline Theme & Quote from diary or interview \\
\hline Challenges in delivering CST-PD (discourse) & $\begin{array}{l}\text { I probably didn't like some of the wording in the, and I can't even pick anything specific. I strug- } \\
\text { gled a bit with some of them and I'd look and think hmmm, it felt a bit stilted with the ques- } \\
\text { tions. [Care partner, CS6, interview] } \\
\text { I wanted to get a discussion going and it didn't come out as a discussion, it was more questioning } \\
\text { and [him] answering. [Care partner, CS17, interview] } \\
\text { Have we done it right? I felt sometimes that maybe I was the problem. Maybe I wasn't approach- } \\
\text { ing this right and I wasn't getting the best out of [him]. [Care partner, CS4, interview] } \\
\text { You'd come to the question and you'd say, 'Well, I've already said that", so what else can I say? } \\
\text { [Participant with PRD, CS5, interview] } \\
\text { I'm thinking 'if this is supposed to last } 30 \text { min there was no way it could have lasted that long' } \\
\text { [...] [the topic] was quite, erm, quite a short one and I'm thinking well how can you flesh that } \\
\text { out? [Care partner, CS6, interview] } \\
\text { I struggled on this topic as I was not familiar with all the seven wonders. [...] I was not able to } \\
\text { give him the support he needed. [Care partner, CS6, diary] } \\
\text { Trying to make it last } 30 \text { min! [Care partner, CS3, diary] } \\
\text { Didn't have much to discuss because of our lack of knowledge. [Care partner, CS5, diary] } \\
\text { Thought he was back at school, didn't like this. [Care partner, CS8, diary] } \\
\text { Just thought it was boring. [Care partner, CS8, diary] }\end{array}$ \\
\hline $\begin{array}{l}\text { Challenges in delivering CST-PD (managing } \\
\text { disease symptoms) }\end{array}$ & $\begin{array}{l}\text { Sometimes I said, I was fine to do it but I know he wouldn't because he was tired [...] so it is } \\
\text { difficult, more than you think. At the beginning I thought " } 3 \text { sessions that's going to be easy" } \\
\text { towards the end it was getting to two. But then I thought we'd rather have two good sessions } \\
\text { rather than fitting it in for the sake of it. [Care partner, CS14, interview] } \\
\text { Took } 35 / 40 \text { min, needs condensing, [he] became restless. [Care partner, CS7, diary] } \\
\text { We had to stop because [name] was "out of it" - not really well, hence only one session this week. } \\
\text { [Care partner, CS11, diary] } \\
\text { [name]'s apparent early loss of interest. [Care partner, CS10, diary] } \\
\text { It highlighted how [name] needs a strong regular interest in a topic to be truly engaged. [Care } \\
\text { partner, CS16, diary] } \\
\text { Needed prompting at times, didn't take the lead role! [Care partner, CS14, diary] } \\
\text { [name] took more interest in the subject today and it was less like "pulling teeth". [Care partner, } \\
\text { CS17, diary] } \\
\text { There were a lot of components to this question, he got tired after the first page. [Care partner, } \\
\text { CS2, interview] } \\
\text { It was quite difficult for the person I look after to understand the task. [Care partner, CS3, diary] } \\
\text { I think it did become tired, didn't it, you know, um, there were occasions where we said, well, I } \\
\text { think we've done enough for today, I think let's finish it off tomorrow. [Participant with PRD, } \\
\text { CS17, interview] } \\
\text { C: Perhaps you get a bit tired. P: Weary. C: Well, it wasn't [...] the project's fault, it was just us } \\
\text { not getting the balance quite right. [Participant with PRD and Care partner, CS5, interview] }\end{array}$ \\
\hline
\end{tabular}

incurs delays; (2) the recruitment target, while achieved, was ambitious, and a rate of one dyad per site per 6 weeks may be more realistic; (3) to account for variations in delivery of the intervention, as well as certain challenges faced by care partners in the role of therapists, inclusion of lay therapists or volunteer therapists should be considered; this notion was raised at a recent post-study Patient and Public Voice (PPV) event and met with general approval from the group; (4) strategies to increase the number of sessions per week from two to three could include more engaging, digitised presentations of the therapy sessions with dashboards to record progress and number of sessions achieved; and (5) certain components involving communication skills or a reliance on motor skills could be modified to obviate these aspects since this proved a challenge for certain participants with communication or physical impairments.

Recruitment was challenging because of the overall frailty of the study population; however, we achieved our threshold for feasibility, eligibility and retention. This was a key finding, particularly since our pre-randomisation refusal of the invitation to participate in the study was high (50\%). It is likely that the relatively high degree of physical frailty of the study population, as well as extent of care partner burden, may have contributed to this high rate of refusal. Nonetheless, our recruitment target was reached since many potential participants, as well as referring clinicians recognised the 
importance of such an intervention, and few opportunities for non-medication-based interventions for people with PD-MCI/PDD/DLB exist. Additionally, most dyads who were initially allocated to the TAU arm expressed interest to receive the intervention following their TAU participation but the decline in their health made them rethink their decision. This highlights the challenges of conducting research in this ever-changing and frail population. Randomisation and blinding were both feasible, although slight imbalance in group characteristics may have been due to the relatively modest number of participant-dyads randomised or the lack of stratification by site. The majority of exploratory assessments were feasible.

The majority of the study design procedures were also feasible. The process evaluation revealed that participantdyads in the CST-PD arm remained in the trial longer than expected due to difficulty in scheduling the therapy training session. A future study could offer a more flexible therapy training option using online or teleconferencing resources.

Regarding intervention fidelity, our quantitative data indicated that care partners had acquired sufficient knowledge to be able to deliver the therapy; however, our qualitative findings suggested some care partners experienced difficulty personalising and adapting the discussion cues provided in the manual. Our findings regarding enactment indicated that additional strategies are needed to boost adherence. One approach to address both of these aspects would be to include a trained, lay therapist to deliver CST-PD. The lay therapist, perhaps a community-based trained volunteer, could visit the participant-dyad once a week to host an additional session. Observing the lay therapist delivering the therapy effectively may increase therapy-delivery efficacy in the care partner through vicarious experience.

The qualitative findings suggested credibility of CST-PD and possible mechanisms by which this type of intervention may be effective: engendering a sense of mastery through implicit cognitive stimulation that builds on retained abilities and social stimulation with the care partner. For example:

"Some of my childhood and things that had gone past, you forgotten all of that you're only assisted, until we sat down in here and we went through the sort of headlines and it was fantastic to thread those paths again and then I can compare with other people's experiences. [Participant with PRD, CS9].

This statement incorporates several potential theoretical mechanisms, both direct and indirect, which are consistent with the multi-faceted nature of the intervention. For example, from the perspective of 'pure CST', change may come about through indirect improvements in cognition resulting from being more relaxed, improved self-esteem due to partner encouragement, greater concentration and memory abilities [32,33]. From the perspective of rehabilitation, change may result from activation of unaffected neural pathways as well as optimisation of remaining abilities, such as in the mechanisms of cognitive rehabilitation therapy applied to dementia [34]. Finally, an important component here is the dyadic aspect and the work undertaken with the spouse, and the person-centred approach resulting from the tailoring of the intervention. The mechanisms underlying dyadic therapies related to improving communication, interpersonal relationships and even intimacy $[35,36]$. Quality of life, as a multi-dimensional outcome of such interventions, will be affected by all these factors: improved cognition and functional ability, reversal of apathy, and improved communication and relationships with care partners [33, 37].

Our study had some limitations that need to be considered. Firstly, since CST-PD was delivered at home by a care partner, we were unable to account in detail for the quality and content of each therapy session, and this is one of the aspects of the intervention that will require modification. Secondly, the imbalance in the number of males compared to female participants may have influenced the reports of acceptability and tolerability of the intervention. This point is important and men with PD are more often studied than women, even out of proportion to the actual prevalence ratio of 2:1; our male: female ratio was still higher at 1:3.75. If studying outcomes such as cognition (e.g., verbal fluency), in which gender differences have been reported [38], such imbalances need to be considered. In addition, there may be some differences in males as compared to females in how PD symptoms affect their lives socially, and this may affect aspects of the intervention. For example, facial masking in women may be contrary to social norms for expressivity in women but not in men, resulting in problems with social relationships for people with PD [39]; however, since our intervention was highly tailored to the individual needs of the participants, such issues and other gender-related aspects are not likely to be significant. Finally, we have found no evidence in the literature of a gender difference in tolerability or acceptability of psychosocial therapies. Stratified randomisation in a future effectiveness trial may preclude such imbalances in baseline characteristics.

In conclusion, our iterative approach to developing and piloting an adapted form of CST is consistent with guidance and represents an important step in the emerging field of psychosocial therapies for this population.

Acknowledgements The authors would like to thank the participants and their care partners for their dedication and commitment to the project. We also acknowledge and thank Martin Orrell and the original CST and iCST development team for giving permission to adapt the therapy, the NIHR for funding the trial, the R\&D personnel involved in study set-up and maintenance, the focus group members who advised on the adaptation of the therapy and, not least, the NHS clinicians and nursing teams involved in referral of participants to the trial, including Patsy Cotton, Carol Miller and Ailish Fountain. We would also like to thank Sophie Baker for her committed work on the project and the 
Clinical Research Network teams in England who helped with recruitment, data collection and trial procedures, including, but not limited to, Heena Mistry, Judith Brooke, Anita Davies, Audrey Williamson, Deborah Risorto, Caroline Cheetham, Emma Eccles, Joanne Woodward, Jane Burgess, Jacki Stansfeld, Katy Aros, Eanna Hanratty and Ritchard Ledgerd.

Author contributions IL conceived and was Chief Investigator of the INVEST study. IL, SAM, SV, SJS, VO and EP were involved in the organization and execution of the research project. IL, L-AC contributed to the statistical analysis. All authors were involved in the writing and critical review of the manuscript.

Funding This paper presents independent research funded by the National Institute for Health Research (NIHR) under its Research for Patient Benefit (RfPB) Programme (PB-PG-0613-31058). The trial was sponsored by the Greater Manchester Mental Health NHS Foundation Trust, UK. The views expressed are those of the authors and not necessarily those of the NIHR or the Department of Health and Social Care.

\section{Compliance with ethical standards}

Conflicts of interest VO has a patent: iCST Manual. All other authors declare that they have no conflict of interest.

Ethical standards The study was approved by a local Research Ethics Committee and conducted according to the ethical standards of the Declaration of Helsinki.

Informed consent All participants in the study provided written informed consent prior to entering the study.

Open Access This article is distributed under the terms of the Creative Commons Attribution 4.0 International License (http://creativeco mmons.org/licenses/by/4.0/), which permits unrestricted use, distribution, and reproduction in any medium, provided you give appropriate credit to the original author(s) and the source, provide a link to the Creative Commons license, and indicate if changes were made.

\section{References}

1. Hobson P, Meara J (2015) Mild cognitive impairment in Parkinson's disease and its progression onto dementia: a 16-year outcome evaluation of the Denbighshire cohort. Int J Geriatr Psychiatry 30:1048-1055. https://doi.org/10.1002/gps.4261

2. Savica R, Grossardt BR, Rocca WA, Bower JH (2018) Parkinson disease with and without Dementia: a prevalence study and future projections. Mov Disord 33:537-543. https://doi.org/10.1002/ mds. 27277

3. Kane JPM, Surendranathan A, Bentley A et al (2018) Clinical prevalence of Lewy body dementia. Alzheimers Res Ther 10:19

4. Orrell M, Yates L, Leung P et al. (2017) The impact of individual cognitive stimulation therapy (iCST) on cognition, quality of life, caregiver health, and family relationships in dementia: a randomised controlled trial. PLoS Med 14(3):e1002269. https:// doi.org/10.1186/ISRCTN65945963

5. Clare L, Kudlicka A, Bayer A et al (2017) Goal-oriented cognitive rehabilitation in early-stage Alzheimer's and related dementias: results from a multi-centre, single-blind, randomised controlled trial (the Great Trial). Alzheimer's Dement 13:P899-P900. https ://doi.org/10.1016/j.jalz.2017.07.312
6. Sindhi A, Leroi I (2013) Nonpharmacological therapies for cognitive enhancement in Parkinson's disease: applying old interventions in a new setting? Neurodegener Dis Manag 3:1-9

7. Orgeta V, McDonald KR, Poliakoff E et al (2015) Cognitive training interventions for dementia and mild cognitive impairment in Parkinson's Disease (Protocol). Cochrane Database Syst Rev. https ://doi.org/10.1002/14651858.CD011961. http://www.cochraneli brary.com

8. Farzana F, Sreekanth V, Mohiuddin MK et al (2015) Can individual home-based cognitive stimulation therapy benefit Parkinson's patients with mild to moderate cognitive impairment? Int J Geriatr Psychiatry 30:433-435

9. McCormick SA, McDonald KR, Vatter S et al (2017) Psychosocial therapy for Parkinson's-related dementia: intervention development. Clin Interv Aging. https://doi.org/10.2147/CIA. S143006

10. Craig P, Dieppe P, Macintyre $S$ et al (2008) Developing and evaluating complex interventions: new guidance. BMJ 337:a1655. https ://doi.org/10.1136/bmj.a1655

11. Lichstein KL, Riedel BW, Grieve R (1994) Fair tests of clinical trials: a treatment implementation model. Adv Behav Res Ther 16:1-29. https://doi.org/10.1016/0146-6402(94)90001-9

12. Leroi I, Vatter S, Carter L-A et al (2019) Parkinson's-adapted Cognitive Stimulation Therapy: a pilot randomised controlled clinical trial. Therapeutic Adv Neurological Disorders (in press)

13. Mccormick SA, Mcdonald KR, Vatter S et al (2017) Psychosocial therapy for Parkinson's-related dementia: study protocol for the INVEST randomised controlled trial. BMJ Open. https://doi. org/10.1136/bmjopen-2017-016801

14. Moher D, Schulz KF, Altman DG (2001) The CONSORT statement: revised recommendations for improving the quality of reports of parallel-group randomised trials. Lancet 357:11911194. https://doi.org/10.1016/S0140-6736(00)04337-3

15. Litvan I, Goldman JG, Tröster AI et al (2012) Diagnostic criteria for mild cognitive impairment in Parkinson's disease: movement disorder society task force guidelines. Mov Disord 27:349-356. https://doi.org/10.1002/mds.24893

16. Emre M, Aarsland D, Brown R et al (2007) Clinical diagnostic criteria for dementia associated with Parkinson's disease. Mov Disord 22:1689-1707. https://doi.org/10.1002/mds.21507

17. McKeith IG, Boeve BF, Dickson DW et al (2017) Diagnosis and management of dementia with Lewy bodies: fourth consensus report of the DLB consortium. Neurology 89:88-100. https://doi. org/10.1212/WNL.0000000000004058

18. Fahn S, Elton R (1987) Recent developments in Parkinson's disease, 2nd edn. Macmilla Health Care Information, Florham Park

19. Schwab RS, England AC (1969) Projection technique for evaluating surgery in Parkinson's disease. In: 3rd symposium on Parkinson's disease, Edinburgh, Livingstone

20. Nasreddine Z, Phillips N, Bédirian V et al (2005) The Montreal Cognitive Assessment, MoCA: a brief screening tool for mild cognitive impairment. J Am Geriatr Soc 53:695-699. https://doi. org/10.1111/j.1532-5415.2005.53221.x

21. Charlesworth G, Burnell K, Hoe J et al (2013) Acceptance checklist for clinical effectiveness pilot trials: a systematic approach. BMC Med Res Methodol. https://doi. org/10.1186/1471-2288-13-78

22. Thabane L, Hopewell S, Lancaster GA et al (2016) Methods and processes for development of a CONSORT extension for reporting pilot randomized controlled trials. Pilot Feasibility Stud 2:1-13. https://doi.org/10.1186/s40814-016-0065-z

23. Orsmond GI, Cohn ES (2015) The distinctive features of a feasibility study: objectives and guiding questions. OTJR Occup Particip Health 35:169-177. https://doi.org/10.1177/1539449215 578649 
24. Sekhon M, Cartwright M, Francis JJ (2017) Acceptability of healthcare interventions: an overview of reviews and development of a theoretical framework. BMC Health Serv Res 17:1-13. https://doi.org/10.1186/s12913-017-2031-8

25. Stanniland C, Taylor D (2000) Tolerability of atypical antipsychotics. Drug Saf 22:195-214

26. Guest G, Bunce A, Johnson L (2006) How many interviews are enough? An experiment with data saturation and variability. Field Methods 18:59-82

27. Julious SA (2005) Sample size of 12 per group rule of thumb for a pilot study. Pharm Stat 4:287-291. https://doi.org/10.1002/pst.185

28. Onder G, Zanetti O, Giacobini E et al (2005) Reality orientation therapy combined with cholinesterase inhibitors in Alzheimer's disease: randomised controlled trial. Br J Psychiatry 187:450-455. https://doi.org/10.1192/bjp.187.5.450

29. Braun V, Clarke V (2006) Using thematic analysis in psychology. Qual Res Psychol 3:77-101

30. Kiresuk TJ, Sherman RE (1968) Goal attainment scaling: a general method for evaluating comprehensive community mental health programs. Community Ment Health J 4:443-453. https:// doi.org/10.1007/BF01530764

31. Hsieh S, Schubert S, Hoon C et al (2013) Validation of the Addenbrooke's cognitive examination III in frontotemporal dementia and Alzheimer's disease. Dement Geriatr Cogn Disord 36:242-250. https://doi.org/10.1159/000351671

32. Spector A, Gardner C, Orrell M (2011) The impact of cognitive stimulation therapy groups on people with dementia: views from participants, their carers and group facilitators. Aging Ment Health 15:945-949

33. Woods B, Thorgrimsen L, Spector A et al (2006) Improved quality of life and cognitive stimulation therapy in dementia. Aging Ment Health 10:219-226

34. Bahar-Fuchs A, Clare L, Woods B (2013) Cognitive training and cognitive rehabilitation for persons with mild to moderate dementia of the Alzheimer's or vascular type: a review. Alzheimers Res Ther 5:35

35. Davies HD, Newkirk LA, Pitts CB et al (2010) The impact of dementia and mild memory impairment (MMI) on intimacy and sexuality in spousal relationships. Int Psychogeriatr 22:618-628

36. Regan TW, Lambert SD, Girgis A et al (2012) Do couple-based interventions make a difference for couples affected by cancer? a systematic review. BMC Cancer 12:279

37. Yates LA, Leung P, Orgeta V et al (2015) The development of individual cognitive stimulation therapy (iCST) for dementia. Clin Interv Aging 10:95-104

38. Weiss EM, Ragland JD, Brensinger CM et al (2006) Sex differences in clustering and switching in verbal fluency tasks. J Int Neuropsychol Soc 12:502-509

39. Hemmesch AR, Tickle-Degnen L, Zebrowitz LA (2009) The influence of facial masking and sex on older adults' impressions of individuals with Parkinson's disease. Psychol Aging 24:542-549

\section{Affiliations}

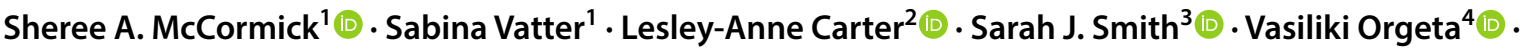 Ellen Poliakoff ${ }^{1}$ (I) Monty A. Silverdale ${ }^{5}$ (D) Jason Raw ${ }^{6} \cdot$ David J. Ahearn $^{7} \cdot$ Christine Taylor $^{8} \cdot$ Joanne Rodda $^{9}$. Tarek Abdel-Ghany ${ }^{10} \cdot$ Benjamin Kwapong $^{1} \cdot$ Iracema Leroi $^{1,11,12}$}

1 Division of Neuroscience and Experimental Psychology, University of Manchester, Jean McFarlane Building, Oxford Road, Manchester M13 9PL, UK

2 Division of Population Health, Health Services Research \& Primary Care, University of Manchester, Manchester, UK

3 School of Health and Community Studies, Leeds Beckett University, Leeds, UK

4 Division of Psychiatry, University College London, London, UK

5 Salford Royal NHS Foundation Trust, Salford, UK

6 Pennine Acute Hospitals NHS Trust, Oldham, UK
7 Manchester University NHS Foundation Trust, Manchester, UK

8 Derbyshire Healthcare NHS Foundation Trust, Derby, UK

9 North East London NHS Foundation Trust, London, UK

10 North West Boroughs Healthcare NHS Foundation Trust, Winwick, UK

11 Greater Manchester Mental Health NHS Foundation Trust, Manchester, UK

12 Global Brain Health Institute, Trinity College Institute of Neuroscience, Trinity College Dublin, Lloyd Building, Dublin 2, Ireland 